Research Article

\section{Innovative results in the treatment of inespecific anusitis-proctitis with the use of bergamot gel (Benebeo gel) ${ }^{\circledR}$}

\author{
Danilo Cafaro ${ }^{1}$, Felipe Celedon ${ }^{1}$, Alessandro Sturiale ${ }^{1}$ and \\ Stefania Sinicropi ${ }^{2 *}$ \\ ${ }^{1}$ Proctology and Pelvic Floor Clinical Centre, University Hospital of Pisa, Italy \\ ${ }^{2}$ Department of Pharmacy Health and Nutritional Sciences, University of Calabria, Italy
}

\section{Introduction}

Inflammation is a complex biological reaction induced by the alteration of tissue homeostasis, which occurs in response to the presence of a biological, chemical or physical agent in the body [1]. The acute inflammatory response is composed of an elaborate cascade of both proinflammatory and anti-inflammatory mediators, and balance between these mediators often determines the outcome after injury [2]. Generally during acute inflammation, cellular and molecular events and interactions reduce the risk of eventual injuries or infections. However, acute inflammation can become chronic, contributing to a variety of chronic inflammatory diseases [3]. Major micro circulatory events that occur during the inflammatory process include changes in vascular permeability, leukocyte recruitment and accumulation, and inflammatory mediator's release [4].

Different classes of medications are used, including corticosteroids, nonsteroidal anti-inflammatory drugs (NSAIDs), and biologic drugs to treat inflammatory disorders. However, they have different side effects, and biologics are particularly expensive. To limit these inconvenients in the last years, the use of complementary anti-inflammatory medicine has increased considerably, including Bergamot, Curcumin, Arnica as many others $[5,6]$. Currently around $80 \%$ of people worldwide use natural products for the prevention and treatment of many diseases, mainly due to their relative safety, effectiveness and low cost. In this field, natural products offer great hope in the identification of bioactive molecules useful for the treatment of inflammatory diseases. Polyphenols, flavonoids, lignans, chloroglucinols, quinones, stilbenes, phenylpropanoids and diarylheptanoids are a very important category of natural compounds capable of modulating inflammatory pathways $[7,8]$.

Anitis is an inflammation of the anal and perianal zone, and proctitis involve the lining of the rectum respectively. Despite

\section{Citrus bergamia}

\section{More Information}

*Address for Correspondence: Stefania Sinicropi, Department of Pharmacy Health and Nutritional Sciences, University of Calabria, Italy, Tel: +39 0984493200; Email: s.sinicropi@unical.it; danilo.cafaro@tiscali.it

Submitted: 19 June 2019

Approved: 26 November 2019 Published: 27 November 2019

How to cite this article: Cafaro D, Celedon F, Sturiale A, Sinicropi S. Innovative results in the treatment of inespecific anusitis-proctitis with the use of bergamot gel (Benebeo gel) ${ }^{\circledR}$. Insights Clin Cell Immunol. 2019; 3: 020-024.

DOI: dx.doi.org/10.29328/journal.icci.1001011

Copyright: (c) 2019 Cafaro D, et al. This is an open access article distributed under the Creative Commons Attribution License, which permits unrestricted use, distribution, and reproduction in any medium, provided the original work is properly cited.

\section{W) Check for updates}

both are frequent clinical conditions, it is not easy to define and sometimes it is not considered in diagnostic therapeutic terms. Anitis/Proctitis would include all inflammatory and infectious lesions of the anus, including cryptitis, papillitis, as well as anal fissures and hemorrhoidal pathology. Chronic nonspecific diarrhea, alterations of the intestinal bacterial flora, antibiotic therapy, parasitosis, perianal erythema, gynecological and urinary infections are elements that should be considered when treating the inflammatory states of the anus and rectum. After excluding infections, malignant lesions [9] and inflammatory bowel disease [10], the main treatment is medical, with the use of phlebotrophic drugs, anti-hemorrhoid or anti-inflammatory topical ointments, selfdilation and application of heat to treat sphincter hypertonia. In some extreme cases, resorting to surgical interventions to treat related pathologies is not always resolutive, and in many cases may be responsible for the persistence and accentuation of the local inflammatory response. We believe that nutraceutical can find a correct and innovative space with excellent clinical results in this pathological field. In this work we provide information about the action of the components of bergamot, and also the use of a gelatinous mixture based on bergamot flavonoids and essential oil used in the treatment of nonspecific proctitis.

The historical information about the methods and times that marked the introduction of bergamot in the province of Reggio Calabria is quite controversial. Among the probable 
places of origin are Greece, Spain and the Canary Islands. This tree has 3 recognized cultivars: Castagnaro, Femminello and Fantastic. The reproduction is done in bitter orange, in the fourth year of sowing, but it is necessary to wait three years for the tree to begin to bear fruit. The main producer of bergamot is Italy. $95 \%$ of the production is cultivated in the coastal strip of the province of Reggio Calabria. The average annual production is $200,000 \mathrm{~kg}$ of fruit.

\section{Phytochemical studies}

On the basis of the previous phytochemical investigations reported in the literature on industrially prepared bergamot juice, it is chemically rich in peculiar constituents such as flavonoids, furanocoumarins, caffeic acid derivatives, sugars and amino acids. Flavonoids are a class of secondary metabolites widespread in the plant kingdom, usually in glycosidate form, which have been identified in bergamot juice as main elements $[11,12]$. In our HPLC chromatographic analysis with mass spectrometry detector (ESI-MS-MS) we identified (Table 1). In the last decade, several researchers have begun to study the biological properties of bergamot derivatives, obtaining important scientific results. Was evaluated for its potential neuroprotection [13], prevention of colorectal cancer [14], antioxidant [15], anti-inflammatory [16] and hypolipidemic drug [17]. Several studies, including ours, have documented the ability of molecules present in bergamot to counteract inflammation through the modulation of different pathways [18-20]. The experiments carried out in our research laboratory showed that some flavonoids (Esperidin, narginine, apigenin, eriotricin) were able to inhibit both gene expression and the pro-inflammatory cytokine secretion. These flavonoids could influence cellular function by acting as activators of SIRT1, a nuclear histone deacetylase involved in the inhibition of the nuclear transcription factor signaling KB (NF-KB). They are also able to inhibit the action of various proinflammatory activators, such as Prostaglandin E2 (PGE2), nitric oxide and reactive nitrogen species.

\section{Benebeo gel ${ }^{\circledR}$ product components}

\section{Bergamot oil and its flavonoids}

Hesperidin: Is one of the characteristic flavonoids of Bergamot where it is concentrated mainly in the skin of the fruit. It has benefits on cutaneous functions, which are attribuited to its antioxidant properties, including

Table 1: HPLC chromatographic analysis with mass spectrometry detector (ESI-MS-MS).

\begin{tabular}{|c|c|c|c|c|}
\hline $\mathbf{R}_{1}$ & $\mathbf{R}_{2}$ & $\mathbf{R}_{3}$ & Struttura & Nome comune \\
\hline O-Rut & $\mathrm{OH}$ & $\mathrm{OH}$ & eriodictiolo 7-O-rutinoside & eriocitrina \\
\hline O-Nh & $\mathrm{OH}$ & $\mathrm{OH}$ & eriodictiolo 7-O-neohesperidoside & neoeriocitrina \\
\hline O-Nh-di-ox & $\mathrm{OH}$ & $\mathrm{OH}$ & & neoeriocitrina-di-ox \\
\hline O-Nh & $\mathrm{H}$ & $\mathrm{OH}$ & $\begin{array}{c}\text { naringenina } \\
\text { 7-O-neohesperidoside }\end{array}$ & naringina \\
\hline O-Nh-di-ox & $\mathrm{H}$ & $\mathrm{OH}$ & & naringina-di-ox \\
\hline O-Nh & $\mathrm{OH}$ & $\mathrm{OMe}$ & esperetina 7-O-neohesperidoside & neoesperidina \\
\hline O-Nh-di-ox & $\mathrm{OMe}$ & $\mathrm{OH}$ & & neoesperidina-di-ox \\
\hline
\end{tabular}

inhibition of microtubule associated protein kinase (MAPK)dependent signaling pathways, and stimulation of epidermal proliferation, differentiation, and lipid production [21]. As anti-inflammatory, it inhibits both gene expression and secretion of LPS-induced pro-inflammatory cytokines (IL6 , IL-1 $\beta$, TNF- $\alpha$ ) by a mechanism involving the inhibition of NF- $\kappa$ B activation [22]. In this same field, there is a metaanalysis which concludes that hesperidin supplementation significantly improves VCAM-1 levels [23].

Narginine: Is a glycoside flavonoid which, in addition to conferring the typical bitter sour taste of bergamot, presents in various in vitro studies and in vivo anti-inflammatory action with a mechanism of inhibition of oxidative stress induced and tissue damage caused by free radicals. A part of these effects is due to the inhibition of myeloperoxidase (MPO) and consequent reduction of the nuclear translocation of NF-KB responsible for the activation of the proinflammatory cytokines [24].

Apigenin: Polyphenol founded in many foods known and studied for its anti-tumor effects [25]. Like most flavonoids, apigenin has anti-inflammatory, antispasmodic and antioxidant properties. It has the ability to inhibit the expression and activation of inflammation mediators at the cellular level, especially interleukins and NF-KB. It also has vascular properties, strengthening the dermal matrix that supports the microvascular network. It inhibits matrix metalloproteases 1 , and reduces their expression by inhibiting the activation of the AP1 protein, reducing the degradation of the collagen of the dermal matrix. This leads to greater resistance and elasticity of the vessel wall [26].

Eriocitrin: It is a flavonoid with different biopharmacological properties, it acts as an epithelising, anti-infective, emollient, anti-edematous, local anesthetic agent. All these effects have been reported in various studies for the treatment of cutaneous wounds and in burns where a capacity to stimulate the processes of granulation and tissue regeneration was observed alongside a decrease in permeability of blood vessels. Another important action of eriotricin and the protective effect against damage caused by free radicals [27]. This reacts against free radicals preventing the alteration of membrane phospholipids and protecting unsaturated fatty acids by blocking peroxidase. It is important to underline that the superoxide radical anion appears to be implicated in the non-enzymatic proteolysis of collagen, so its inhibition effectively protects this important substance. The main effect is given by the Scavinger radical action [28].

\section{Bergamot essential oil}

It comes from the pressing of the peel of the ripe fruit. More and more recent studies have developed in the field of psychiatry as anxiolytic [29,30], and also for its antriproliferative effects [31] and anticancer activity in vitro [32]. And there are studies in mice that suggest that bergamot oil may be relevant in the treatment of vascular disorders in 
which proliferation of smooth muscle cell and oxyLD-related endothelial cell dysfunction are involved [33]. Bergamot essential oil comes from the pressing of the peel of the ripe fruit. Bergamot oil has been used in Italian folk medicine for centuries, especially against fevers and gastro-intestinal problems. Increasingly recent studies by the University of Calabria [34,35], the Universities of Parma, Messina, Tor Vergata and Magna Graecia confirm the innumerable pharmacological and therapeutic properties of oil. Its beneficial effects derive from its chemical composition, from the presence of amino acids, mineral salts, vitamin $\mathrm{C}$ and above all from the high concentration of flavonoid polyphenols. The radical-scavenging and antioxidant action is determined by the inhibition of cellular myeloperoxidase (MPO) activity and by the inhibition of the adhesion molecules ICAM1 and P-selectin involved in inflammatory processes. Furthermore, in our study we observed the ability to reduce NF-KB nuclear translocation and the release of proinflammatory cytokines in the colitis induced in laboratory mice. The nuclear transcription factor NF-KB is the main regulator of the inflammatory response that drives the activation of genes associated with the transcription of inflammatory mediators, such as interleukins, tumor necrosis factor $\alpha$ (TNF $\alpha)$ and prostaglandins (PGs), as well as inflammatory enzymes such as nitric oxide and cyclooxygenases (COX2). Their inhibition is the basis for the reduction of the inflammatory process.

\section{Materials and Methods}

From January 2018 to January 2019, all the patients referred to our clinic with a diagnosis of idiopatic or hemorroids-related anitis and/or proctitis were treated using the local bergamot-derived gel. The bergamot-derived gel (Benebeo gel ${ }^{\circledR}$ ) was used twice per day through the dedicated cannula for 30 days. After the first visit (T0), the follow-up was scheduled as follows: 30 days (T1) and 90 days (T2). All the visit were performed using the Digital Videoproctoscope. The symptoms investigated were pain (VAS scale 0-10), itching, bleeding (absent, mild, intense) and hyperemia (absent, mild, severe). The satisfaction of the patients after medical treatment was measured in a 0-10 scale.

\section{Results}

From January 2018 to January 2019, a total of 178 patients (95 M -83 F) with a mean age of 53 year (range 24-76 y) were treated with the of local bergamot-derived gel. $94 \%$ of the patients reported a symptoms relief at $\mathrm{T} 1$ with a maintained wellbeing at $\mathrm{T} 2$ in $89 \%$. The mean satisfaction rate after the treatment was 8.2 (range 6-10). The mean value of pain VAS scale before treatment was 6 and decrease to 2 after the treatment at T2. The clinical evaluation performed with Digital Video proctoscope showed a decreased of bleeding and the related hyperemia in $78.9 \%$ and $79.6 \%$ of patients respectively (Figure 1). The images of clinical improvement are shown in figure 2.

\section{Discussion}

Inflammation and oxidative stress are pathophysiological events that are closely related to each other. One of them may appear sooner or later, but when one emerges, the other is more likely to follow it and then both are involved in the pathogenesis of many disorders. Just as the inflammatory process can induce oxidative stress, the latter can cause inflammation by activating multiple pathways [36,37]. The result is that both inflammation and oxidative stress are associated with certain chronic diseases. In pathological inflammatory conditions, there may be an exaggerated generation of reactive species that can spread outside the cells, which induces localized oxidative stress and tissue damage. In addition, activated phagocytized cells produce large amounts of radical oxygen and reactive nitrogen species. At the onset of inflammation, infection or tissue damage is perceived by pattern recognition receptors such as Toll-like receptors (TLRs), NOD-type receptors (NLRs) and the receptor for advanced glycation end products (RAGE). The stimulation of these receptors by binding to specific molecules leads to the activation of transcription factors such as the nuclear factor

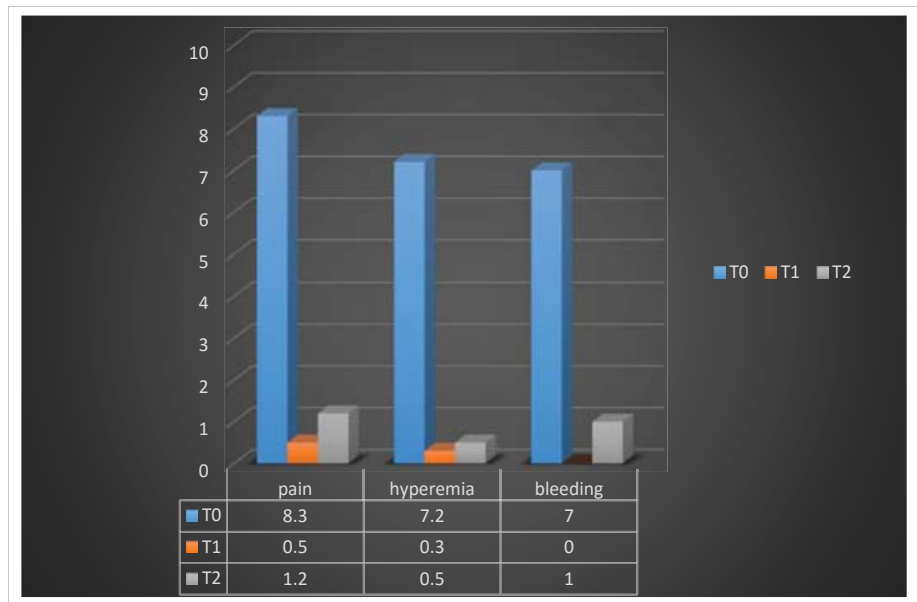

Figure 1: Improvement of bleeding before the treatment after the treatment at 90 days (T2). Improvement of local hyperemia before the treatment after the treatment at 90 days (T2).
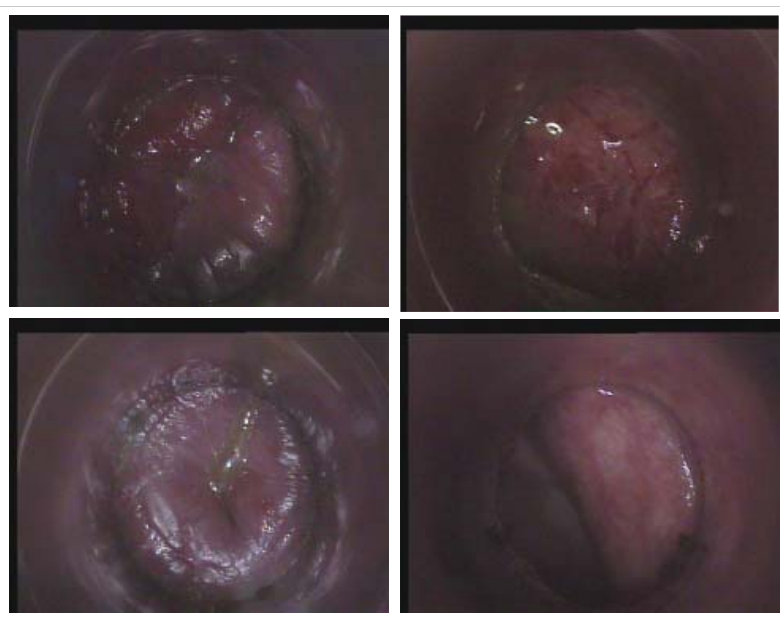

Figure 2: A-B: Ano-proctoscopy before treatment with hyperemia, C-D: Anoproctoscopy after treatment with the resolution of local inflammation. 
KB (NF-KB) and the activation of protein-1 (AP1), which in turn induces the expression of genes proinflammatories, exercising antimicrobial functions and recruiting additional immune cells [38,39]. It has been shown that hydrogen peroxide induces inflammation by activating these same transcription factors. The presence of bioactive molecules capable of blocking inflammatory processes through multiple pathways, both by inhibiting the cellular transcription factor $\mathrm{KB}(\mathrm{NF}-\mathrm{KB})$ as well as the inhibitory and reducing factors of reactive nitrogen species, and through inhibition of proinflammatory drugs such as cyclooxygenases (COX), represent a current pharmacological weapon. In particular, the effect of the fraction of flavonoids isolated by us in association with the essential oil of bergamot leads to an improvement both in the inflammatory situation and in the symptoms. The action of flavonoids extracted from bergamot could reduce logistic processes by reducing intracellular free radicals, in particular the expression of cyclooxygenase-2 (COX2). They could also reduce and modulate the inflammatory response by inhibiting prostaglandins and leukotrienes. A further action of the compound based on flavonoids and essential oil of Bergamot synthesized in this way would be able to block the intracellular receptor expression of NF-KB by inhibiting the mechanisms responsible for the inflammatory cascade.

\section{Conclusion}

Bergamot is used in the cosmetic, pharmaceutical and food industries. The interest and beneficial effects of bergamot juice have been increased by its healing promoting activity. The objective of the study was to evaluate the anti-inflammatory effects of non-specific proctitis. The results of this study suggested that the administration of local bergamot gel had an anti-inflammatory effect with a faster healing process.

\section{References}

1. Maskrey BH, Megson IL, Whitfield PD, Rossi AG. Mechanisms of resolution of inflammation: a focus on cardiovascular disease. Arterioscler Thromb Vasc Biol. 2011; 31: 1001-1006.

PubMed: https://www.ncbi.nlm.nih.gov/pubmed/21508346

2. Ward PA, Lentsch $A B$. The acute inflammatory response and its regulation. Arch Surg. 1999; 134: 666-669.

PubMed: https://www.ncbi.nlm.nih.gov/pubmed/10367878

3. Chen L, Deng H, Cui H, Fang J, Zuo Z, Deng J, Li Y, Wang X, Zhao L. Inflammatory responses and inflammation-associated diseases in organs. Oncotarget. 2017; 9: 7204-7218.

PubMed: https://www.ncbi.nlm.nih.gov/pubmed/29467962

4. Ferrero-Miliani L, Nielsen $\mathrm{OH}$, Andersen PS, Girardin SE. Chronic inflammation: importance of NOD2 and NALP3 in interleukin-1beta generation. Clin Exp Immunol. 2007; 147: 227-235.

5. Kriplani P, Guarve K, Baghel US. Novel Herbal Topical Patch containing Curcumin and Arnica Montana for the Treatment of Osteoarthritis. Curr Rheumatol Rev. 2019.

PubMed: https://www.ncbi.nlm.nih.gov/pubmed/30767746

6. Miller AD, Ly BT, Clark RF. Neonatal hemolysis associated with nursing mother ingestion of arnica tea. Clin Toxicol (Phila). 2009; 47: 726. PubMed: https://www.ncbi.nlm.nih.gov/pubmed/17223962

7. Spigoni V, Mena P, Fantuzzi F, Tassotti M, Brighenti F, et al. Bioavailability of Bergamot (Citrus bergamia) Flavanones and Biological Activity of Their Circulating Metabolites in Human Pro-Angiogenic Cells. Nutrients. 2017; 9: 12

PubMed: https://www.ncbi.nlm.nih.gov/pubmed/29211032

8. FormisanoC,RiganoD,LopatrielloA,SirignanoC,RamaschiG,etal.Detailed Phytochemical Characterization of Bergamot Polyphenolic Fraction (BPF) by UPLC-DAD-MS and LC-NMR. J Agric Food Chem. 2019; 67: 11. PubMed: https://www.ncbi.nlm.nih.gov/pubmed/30807134

9. Penninger JL, Zainea GG. Common anorectal conditions: Part II. Lesions. Am Fam Physician. 2001; 64: 77-88.

PubMed: https://www.ncbi.nlm.nih.gov/pubmed/11456437

10. Dawson H, Serra S. Tumours and inflammatory lesions of the anal canal and perianal skin revisited: an update and practical approach. $\mathrm{J}$ Clin Pathol; 68: 971-981.

PubMed: https://www.ncbi.nlm.nih.gov/pubmed/26602415

11. Gattuso G, Caristi C, Gargiulli C, Bellocco E, Toscano G, et al. Flavonoid glycosides in bergamot juice (Citrus bergamia Risso). J Agric Food Chem. 2006; 54: 3929-3935.

PubMed: https://www.ncbi.nlm.nih.gov/pubmed/16719517

12. Dugo $P$, Presti ML, Ohman M, Fazio A, Dugo G, et al. Determination of flavonoids in citrus juices by micro-HPLC-ESI/MS. J Sep Sci. 2005; 28 : 1149-1156.

PubMed: https://www.ncbi.nlm.nih.gov/pubmed/16116991

13. Corasaniti MT, Maiuolo J, Maida S, Fratto V, Navarra M, et al. Cell signaling pathways in the mechanisms of neuroprotection afforded by bergamot essential oil against NMDA-induced cell death in vitro. Br J Pharmacol. 2007; 151: 518-529.

PubMed: https://www.ncbi.nlm.nih.gov/pubmed/17401440

14. Navarra M, Femia AP, Romagnoli A, Tortora K, Luceri C, et al. A flavonoid-rich extract from bergamot juice prevents carcinogenesis in a genetic model of colorectal cancer, the Pirc rat (F344/NTacApcam1137). Eur J Nutr. 2019.

PubMed: https://www.ncbi.nlm.nih.gov/pubmed/30919084

15. La Russa D, Giordano F, Marrone A, Parafati M, Janda $E$, et al. Oxidative Imbalance and Kidney Damage in Cafeteria DietInduced Rat Model of Metabolic Syndrome: Effect of Bergamot Polyphenolic Fraction. Antioxidants (Basel). 2019; 8.

PubMed: https://www.ncbi.nlm.nih.gov/pubmed/30884780

16. Perna S, Spadaccini D, Botteri L, Girometta C, Riva A, et al. Efficacy of bergamot: From anti-inflammatory and anti-oxidative mechanisms to clinical applications as preventive agent for cardiovascular morbidity, skin diseases, and mood alterations. Food Sci Nutr. 2019; 7: 369-384. PubMed: https://www.ncbi.nlm.nih.gov/pubmed/30847114

17. Mirielle CN, Jeremy JJ. Clinical application of bergamot (Citrus bergamia) for reducing high cholesterol and cardiovascular disease markers. Integr Food Nutr Metab. 2019.

PubMed: https://www.ncbi.nlm.nih.gov/pubmed/31057945

18. Parafati M, Lascala A, La Russa D, Mignogna C, Trimboli F, et al. Bergamot Polyphenols Boost Therapeutic Effects of the Diet on NonAlcoholic Steatohepatitis (NASH) Induced by "Junk Food": Evidence for Anti-Inflammatory Activity. Nutrients. 2018; 10.

PubMed: https://www.ncbi.nlm.nih.gov/pubmed/30388763

19. Daniela I, Marika C, Michela C, Enrico G, Emanuela E, et al. Antiinflammatory and Antioxidant Effects of Flavonoid-Rich Fraction of Bergamot Juice (BJe) in a Mouse Model of Intestinal Ischemia/ Reperfusion Injury. Front Pharmacol. 2016; 7: 203.

PubMed: https://www.ncbi.nlm.nih.gov/pubmed/27471464

20. Impellizzeri D, Bruschetta G, Di Paola R, Ahmad A, Campolo $M$, et al. The anti-inflammatory and antioxidant effects of bergamot juice extract (BJe) in an experimental model of inflammatory bowel disease. Clin Nutr. 2015; 34: 1146-1154. PubMed: https://www.ncbi.nlm.nih.gov/pubmed/25491246

21. Man MQ, Yang B, Elias PM. Benefits of Hesperidin for Cutaneous Functions. Evid Based Complement Alternat Med. 2019; 2019: 
2676307.

PubMed: https://www.ncbi.nlm.nih.gov/pubmed/31061668

22. Risitano R, Currò M, Cirmi S, Ferlazzo N, Campiglia P, et al. Flavonoid fraction of Bergamot juice reduces LPS-induced inflammatory response through SIRT1-mediated NF-KB inhibition in THP-1 monocytes. PLoS One. 2014; 9: e107431.

PubMed: https://www.ncbi.nlm.nih.gov/pubmed/25260046

23. Lorzadeh E, Ramezani-Jolfaie N, Mohammadi M, Khoshbakht Y, SalehiAbargouei A. The effect of hesperidin supplementation on inflammatory markers in human adults: A systematic review and meta-analysis of randomized controlled clinical trials. Chem Biol Interact. 2019;307: 8-15. PubMed: https://www.ncbi.nlm.nih.gov/pubmed/30991044

24. Nisticò S, Ehrlich J, Gliozzi M, Maiuolo J, Del Duca E, et al. Telomere And Telomerase Modulation By Bergamot Polyphenolic Fraction In Experimental Photoageing In Human Keratinocytes. J Biol Regul Homeost Agents. 2015; 29: 723-728.

PubMed: https://www.ncbi.nlm.nih.gov/pubmed/26403416

25. Ittiudomrak T, Puthong S, Roytrakul S, Chanchao C. 15a-Mangostin and Apigenin Induced Cell Cycle Arrest and Programmed Cell Death in SKOV-3 Ovarian Cancer Cells. Toxicol Res. 2019; 35: 167-179.

PubMed: https://www.ncbi.nlm.nih.gov/pubmed/31015899

26. Graziano AC, Cardile V, Crascì L, Caggia $S$, Dugo $P$, et al. Protective effects of an extract from Citrus bergamia against inflammatory injury in interferon-y and histamine exposed human keratinocytes. Life Sci. 2012; 90: 968-974.

PubMed: https://www.ncbi.nlm.nih.gov/pubmed/22634580

27. Hiramitsu $M$, Shimada $Y$, Kuroyanagi J, Inoue $T$, Katagiri $T$, et al. Eriocitrin ameliorates diet-induced hepatic steatosis with activation of mitochondrial biogenesis. Sci Rep. 2014; 4: 3708.

PubMed: https://www.ncbi.nlm.nih.gov/pubmed/24424211

28. Miyake Y. Lemon flavonoid, eriocitrin, suppresses exercise-induced oxidative damage in rat liver. Life Sciences.

29. Bruno A, Pandolfo G, Crucitti M, Cedro C, Zoccali RA, et al. Bergamot Polyphenolic Fraction Supplementation Improves Cognitive Functioning in Schizophrenia: Data From an 8-Week, Open-Label Pilot Study. J Clin Psychopharmacol. 2017; 37: 468-471.

PubMed: https://www.ncbi.nlm.nih.gov/pubmed/28591067

30. Rombolà L, Tridico L, Scuteri D, Sakurada T, Sakurada S, et al.
Bergamot Essential Oil Attenuates Anxiety-Like Behaviour in Rats. Molecules. 2017; 11: 22.

PubMed: https://www.ncbi.nlm.nih.gov/pubmed/28398260

31. Navarra M, Ferlazzo N, Cirmi S, Trapasso E, Bramanti P, et al. Effects of bergamot essential oil and its extractive fractions on $\mathrm{SH}-\mathrm{SY} 5 \mathrm{Y}$ human neuroblastoma cell growth. J Pharm Pharmacol. 2015; 67: 1042-1053. PubMed: https://www.ncbi.nlm.nih.gov/pubmed/25808679

32. Celia C, Trapasso E, Locatelli $M$, Navarra $M$, Ventura $C A$, et al. Anticancer activity of liposomal bergamot essential oil (BEO) on human neuroblastoma cells. Colloids Surf B Biointerfaces. 2013. PubMed: https://www.ncbi.nlm.nih.gov/pubmed/24099646

33. Danilo Cafaro; Maria Stefania Sinicropi. Creation and experimentation of mesalazine gel and bergamot flavonoid fraction (Benebeogel ${ }^{\circledR}$ ) for the treatment ofnon-specific proctitis. Tech Coloproctol. 2018.

34. Cafaro D, Sturiale A, Fortuna V, Sinicropi MS' Naldini G. Clinical effect of the bergamot-derived gel (Benebeo gel $\circledast$ ) associated with mesalazine for the treatment of anitis and proctitis. Trend Gen Pract. 2018; 2.

35. Shanley TP, Foreback JL, Remick DG, Ulich TR, Kunkel SL, et al. Regulatory effects of interleukin- 6 in immunoglobulin $\mathrm{G}$ immune complex-induced lung injury. Am J Pathol. 1997; 151: 193- 203. PubMed: https://www.ncbi.nlm.nih.gov/pubmed/9212745

36. Castellani P, Balza E, Rubartelli A. Inflammation, DAMPs, Tumor Development, and Progression: A Vicious Circle Orchestrated by Redox Signaling. Antioxid. Redox Signal. 2014; 20: 1086-1097.

PubMed: https://www.ncbi.nlm.nih.gov/pubmed/23373831

37. Tabas I, Glass CK. Anti-inflammatory therapy in chronic disease: Challenges and opportunities. Science. 2013, 339: 166-172. PubMed: https://www.ncbi.nlm.nih.gov/pubmed/23307734

38. Oliveira-Marques V, Marinho HS, Cyrne L, Antunes F. Role of hydrogen peroxide in NF-kappaB. Activation: From inducer to modulator. Antioxid Redox Signal. 2009, 11, 2223-2243.

PubMed: https://www.ncbi.nlm.nih.gov/pubmed/19496701

39. Vollgraf, U, Wegner M, Richter-Landsberg C. Activation of AP-1 and nuclear factor-kappaB transcription factors is involved in hydrogen peroxide-induced apoptotic cell death of oligodendrocytes. J. Neurochem. 1999; 73: 2501-2509.

PubMed: https://www.ncbi.nlm.nih.gov/pubmed/10582611 